\title{
Thermodynamics of a Colloidal Particle in a Time-Dependent Nonharmonic Potential
}

\author{
V. Blickle, ${ }^{1}$ T. Speck, ${ }^{2}$ L. Helden, ${ }^{1}$ U. Seifert, ${ }^{2}$ and C. Bechinger ${ }^{1}$ \\ ${ }^{1}$ 2. Physikalisches Institut, Universität Stuttgart, Pfaffenwaldring 57, 70550 Stuttgart, Germany \\ ${ }^{2}$ II. Institut für Theoretische Physik, Universität Stuttgart, Pfaffenwaldring 57, 70550 Stuttgart, Germany
}

(Received 21 November 2005; published 23 February 2006)

\begin{abstract}
We study the motion of an overdamped colloidal particle in a time-dependent nonharmonic potential. We demonstrate the first lawlike balance between applied work, exchanged heat, and internal energy on the level of a single trajectory. The observed distribution of applied work is distinctly non-Gaussian in good agreement with numerical calculations. Both the Jarzynski relation and a detailed fluctuation theorem are verified with good accuracy.
\end{abstract}

DOI: 10.1103/PhysRevLett.96.070603

PACS numbers: 05.40.-a, 05.70.-a, 82.70.Dd

Since more than a century, the first law relating the work applied to a system with both the exchanged heat and an increase in internal energy is one of the cornerstones of macroscopic physics. Its consistent formulation for a mesoscopic system like a driven colloidal particle, however, was suggested only about a decade ago [1]. Since on these scales thermal fluctuations are relevant, probability distributions for work, heat, and internal energy replace the sharp values of their macroscopic counterparts. Various theoretical relations like the fluctuation theorem [2,3], the Jarzynski relation [4], and the Hatano-Sasa relation [5] involving these distributions in different settings extend the second law to the mesoscopic realm at least as long as the notion of a constant temperature of the ambient heat bath remains meaningful [for a review, see [6]]. Such theorems have been tested experimentally using both biomolecules manipulated mechanically $[7,8]$ as well as colloidal particles in time dependent laser traps [9-11]. Common to all colloidal experiments, so far, is that these laser traps generate a harmonic potential albeit with a timedependent center or "spring constant." Consequently, often the interesting distributions are Gaussian even though for certain quantities non-Gaussian distributions can occur $[10,12]$.

In this Letter, we study the thermodynamics of single colloidal trajectories in a time-dependent nonharmonic potential which, generically, gives rise to non-Gaussian distributions. Only for very short or very long trajectories, one expects Gaussian distributions even in this nonharmonic case [13]. In particular, we identify applied work, exchanged heat, and change in internal energy along a single trajectory and thus test the consistency of these notions on this level, or, put differently, illustrate the validity of the first law. We measure the distribution of work in the non-Gaussian regime and compare it to theoretical prediction. Such a comparison does not involve a single fit parameter since all quantities are measured independently, which is another advantage of colloidal systems. Finally, we test the Jarzynski relation which expresses the free energy difference between two equilib- rium states in terms of the nonequilibrium work spent in the transition between the two states. Such an illustration of the Jarzynski relation in the non-Gaussian regime comes timely given ongoing theoretical criticism of its validity [14,15].

In our study, particle trajectories were determined using total internal reflection microscopy (TIRM), where a single colloidal particle is illuminated under evanescent field conditions. This field is created by total internal reflection of a laser beam at a glass-water interface. The scattered intensity of a bead near the interface is proportional to $\exp (-\zeta z)$, with $\zeta^{-1}$ the decay length of the evanescent field and $z$ the particle-wall distance [16]. Measuring the scattered intensity of a fluctuating Brownian particle as a function of time thus yields its vertical position with a spatial resolution of about $5 \mathrm{~nm}$.

We used an aqueous suspension of highly charged polystyrene beads with radius $R=2 \mu \mathrm{m}$, which were illuminated with light of wavelength $\lambda=658.5 \mathrm{~nm}$. The particle concentration was sufficiently low to guarantee that there was only a single particle within the field of view. The penetration depth was adjusted to $\zeta^{-1} \simeq 200 \mathrm{~nm}$ and the scattered intensity was monitored with a photomultiplier at a data acquisition rate of $\nu=2 \mathrm{kHz}$. An additional focused laser beam $(\lambda=1064 \mathrm{~nm}$, power $P \simeq 2 \mathrm{~mW})$ was directed vertically from the top, which confined the particle motion to an one-dimensional trajectory in $z$ direction.

To drive the colloidal particle between two equilibrium states, it was subjected to the light pressure of another optical tweezers $(\lambda=532 \mathrm{~nm}$, beam waist about $17 \mu \mathrm{m}$, $P \leq 60 \mathrm{~mW}$ ), which was incident from below into the sample cell (lower tweezers, see inset of Fig. 1). The intensity of this laser beam was varied with an electrooptical modulator connected to a computer-controlled waveform generator. We modulated the laser intensity according to a time-dependent symmetric protocol $I(\tau)=$ $I\left(t_{\mathrm{s}}-\tau\right)$ in the time interval $0 \leq \tau \leq t_{\mathrm{s}}$, where $t_{\mathrm{s}}$ is the pulse duration (see Fig. 2) [17]. To ensure that the system is out of equilibrium, $t_{\mathrm{s}}$ must be smaller than the particle relaxation time $t_{\mathrm{r}} \simeq 480 \mathrm{~ms}$. On the other hand, when 


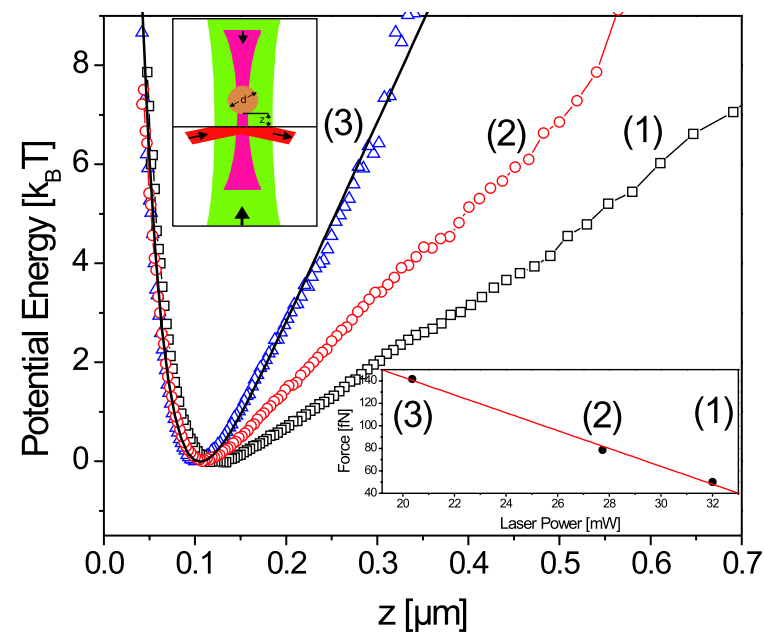

FIG. 1 (color online). Particle-wall interaction potentials for three different intensities of the lower optical tweezers [decreasing power from (1) to (3)]. The solid line shows the fit according to Eq. (1) with $\kappa^{-1} \approx 25 \mathrm{~nm}$. Inset: light pressure vs tweezers intensity. The light pressure is a linear function of the laser intensity.

repeating the experiment the pause $t_{\mathrm{p}}$ between two consecutive pulses must be longer than $t_{\mathrm{r}}$ to guarantee equilibration of the system. To meet both conditions in our experiments we have chosen $t_{\mathrm{s}}=120 \mathrm{~ms}$ and $t_{\mathrm{p}}=$ $700 \mathrm{~ms}$.

The total time-dependent potential acting on the particle at distance $z$ is given by [16]

$$
V(z, \tau)=A_{0} \exp (-\kappa z)+B_{0} z+C_{0} I(\tau) z .
$$

The first term describes the double-layer interaction between the negatively charged colloidal particle and the likely charged wall with $A_{0}$ depending on the corresponding surface charges and $\kappa^{-1}$ the Debye screening length, which depends on the salt concentration in the suspension. The second term accounts for the weight of the particle and the additionally exerted light pressure from the upper tweezers, which both depend linearly on the particle distance $z[18,19]$. The last term considers the timedependent optical forces induced by the lower tweezers. Experimentally, particle-wall potentials are easily obtained by measuring the distance probability distribution $p(z)$ of a colloid in front of a wall. In thermal equilibrium, i.e., for $I(\tau)=$ const, the potentials are given by $V(z)=$ $-k_{\mathrm{B}} T \ln p(z)$ up to a constant. Here, $T$ is the temperature of the environment and $k_{\mathrm{B}}$ is the Boltzmann constant. The symbols in Fig. 1 show $V(z)$ obtained for three different intensities of the lower tweezers. The solid line is a fit to Eq. (1) (exemplarily shown only for one data set) and clearly demonstrates that the particle is moving in a nonharmonic potential. It can be seen in Fig. 1 that the light pressure of the lower tweezers reduces the slope of the linear part of the potential, as already demonstrated by other authors $[16,18]$. The inset of Fig. 1 shows the ex- pected linear dependence on the intensity of the light pressure.

Figure 2 shows exemplarily the trajectory of a particle driven by the time-dependent potential (1). While during the first pulse the particle is strongly forced towards the surface, thermal fluctuations support it to move against the applied force away from the wall during the second pulse. This clearly demonstrates that the particle is strongly coupled to the surrounding heat bath.

In contrast to recent experimental studies [7-11], we want to test experimentally to which precision energy conservation of the particle on its trajectory is maintained. This does not only provide a rigorous check of the experimental technique, data analysis, and the energy resolution but also demonstrates the interplay of applied work and exchanged heat when the system is nonadiabatically driven. Therefore, in addition to the work $W$ exerted on the particle we need to determine its heat exchange $Q$ with the environment.

The Brownian motion of the colloidal bead is described by the Langevin equation

$$
\gamma \dot{z}=-\frac{\partial V}{\partial z}+\xi
$$

with $\gamma$ the friction coefficient and $\xi$ the stochastic force. According to [1] the incremental change of heat $d Q$ and work $d W$ is then given by

$$
d W=\frac{\partial V}{\partial \tau} d \tau, \quad d Q=-\frac{\partial V}{\partial z} d z
$$

Integration along a single trajectory $z(\tau)$ then leads to the work functional

$$
W[z(\tau)]=\int_{0}^{t_{\mathrm{s}}} d \tau \frac{\partial V}{\partial \tau}(z(\tau), \tau)=\frac{C_{0}}{\nu} \sum_{i} \dot{I}\left(\tau_{i}\right) z\left(\tau_{i}\right),
$$

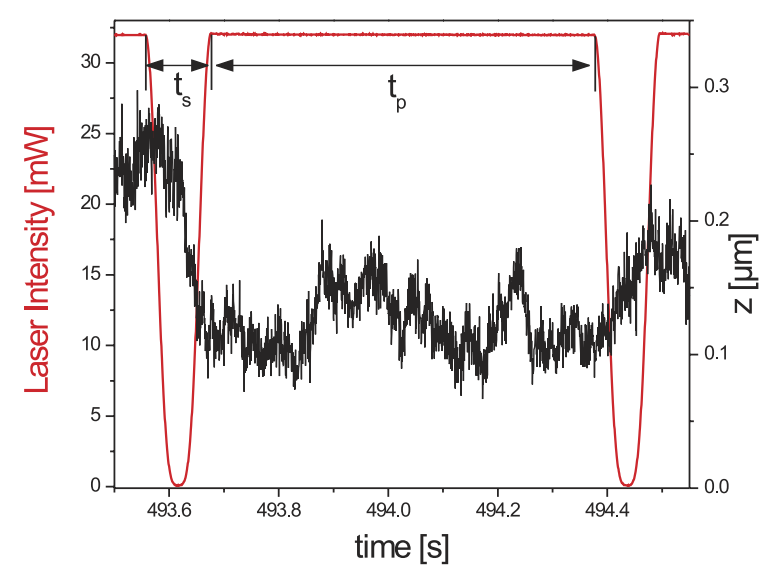

FIG. 2 (color online). Measured tweezers intensity and particle trajectory. During the first pulse the particle is pressed towards the surface. During the second pulse thermal fluctuations support the particle and it is able to move away from the wall. Hence the applied work is positive for the first pulse and negative for the second. 
where we have used Eq. (1). The right-hand side of Eq. (4) accounts for the discrete sampling of the particle trajectory during our experiments with rate $\nu=\frac{1}{\delta t}$ at times $\tau_{i}=i \delta t$. Because the velocity autocorrelation of a Brownian particle decays on a time scale of some $10 \mathrm{~ns}$, the velocity

$$
\overline{\dot{z}}\left(\tau_{i}\right)=\nu \int_{\tau_{i}}^{\tau_{i+1}} d \tau \dot{z}(\tau)
$$

determined from a trajectory measured with $\nu=2 \mathrm{kHz}$ is not identical to the instant particle velocity $\dot{z}$. However, since $\partial V / \partial z$ varies on a time scale much larger than $\delta t$, the heat along a single trajectory $z(\tau)$ can be written as

$$
\begin{aligned}
Q[z(\tau)] & =-\int_{0}^{t_{\mathrm{s}}} d \tau \frac{\partial V}{\partial z}(z(\tau), \tau) \dot{z}(\tau) \\
& =-\frac{1}{\nu} \sum_{i} \frac{\partial V}{\partial z}\left(z\left(\tau_{i}\right), \tau_{i}\right) \overline{\dot{z}}\left(\tau_{i}\right) .
\end{aligned}
$$

With the above sign convention the heat $Q$ is negative (positive) when extracted (delivered) from (to) the thermal environment. Since we have full knowledge of the time dependence of $I, V$, and $z$, both quantities $W$ and $Q$ can be determined from our experiments.

Introducing $\Delta V=V\left(z\left(t_{\mathrm{s}}\right), t_{\mathrm{s}}\right)-V(z(0), 0)$ we finally obtain a stochastic version

$$
W[z(\tau)]-Q[z(\tau)]-\Delta V=0
$$

of the first law of thermodynamics. Figure 3(a) shows work $W$, heat $Q$, and change of inner energy $\Delta V$ for the trajectory of a single particle where the protocol $I(\tau)$ was repeated about 100 times. For $W$ and $Q$ maximal energies of about $15 k_{\mathrm{B}} T$ are observed, whereas $\Delta V$ is on the order of a few $k_{\mathrm{B}} T$. Obviously, $Q$ and $W$ are not independent quantities. Usually trajectories resulting in a large work $W$ are also accompanied by a large value of $Q$. But only when taking all three energies in Eq. (7) into consideration, the distribution of the deviation shown in Fig. 3(b) is centered around zero, having a half width of about $0.7 k_{\mathrm{B}} T$. Assuming that the three terms have the same contribution

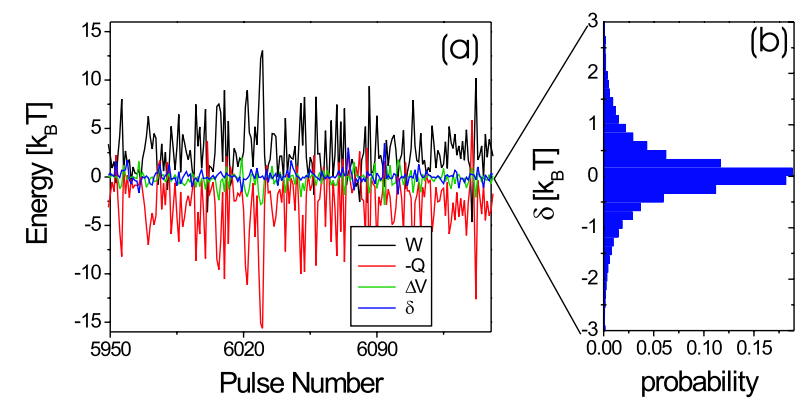

FIG. 3 (color online). (a) The quantities $-Q, W$, and $\Delta V$ for about 100 periods of the protocol $I(\tau)$. (b) Distribution histogram of $\delta=W-Q-\Delta V$, the experimentally observed "deviation" from the first law of thermodynamics. to the total error, the energy error of these energies is about one quarter of $k_{\mathrm{B}} T$.

The measured work distribution in Fig. 4 is distinctly non-Gaussian and exhibits an asymmetry towards larger work values. Whereas the first law is valid along a single trajectory as demonstrated, fluctuation theorems considering probability distributions can be regarded as an extension of the second law. This becomes evident when looking at the Jarzynski relation [4]

$$
\left\langle e^{-W / k_{\mathrm{B}} T}\right\rangle=e^{-\Delta F / k_{\mathrm{B}} T},
$$

where $\Delta F$ is the change of free energy between two equilibrium states and the brackets $\langle\cdots\rangle$ represent the average over the work distribution spent in a transition between these states. Equation (8) immediately leads to $\langle W\rangle \geq \Delta F$, a formulation of the second law for driven systems on the mesoscopic scale. A test of the Jarzynski relation using the data shown in Fig. 4 yields $\left\langle\exp \left(-W / k_{\mathrm{B}} T\right)\right\rangle \simeq 1.03$ in agreement with $\Delta F=0$ for the symmetric protocol $I(\tau)$. In addition to the integral theorem (8) we also test the somewhat stronger detailed fluctuation theorem

$$
P(-W) / P(+W)=e^{-W / k_{\mathrm{B}} T},
$$

which holds for time-symmetric protocols $I(\tau)=I\left(t_{\mathrm{s}}-\tau\right)$ (see inset of Fig. 4) [20,21]. Here the probability $P(-W)$ that a negative work value occurs is compared to the probability $P(+W)$ of a positive value of same magnitude.

In order to compare the measured histogram in Fig. 4 to the theoretical prediction we calculate the probability distribution solving the Fokker-Planck equation $[13,22]$

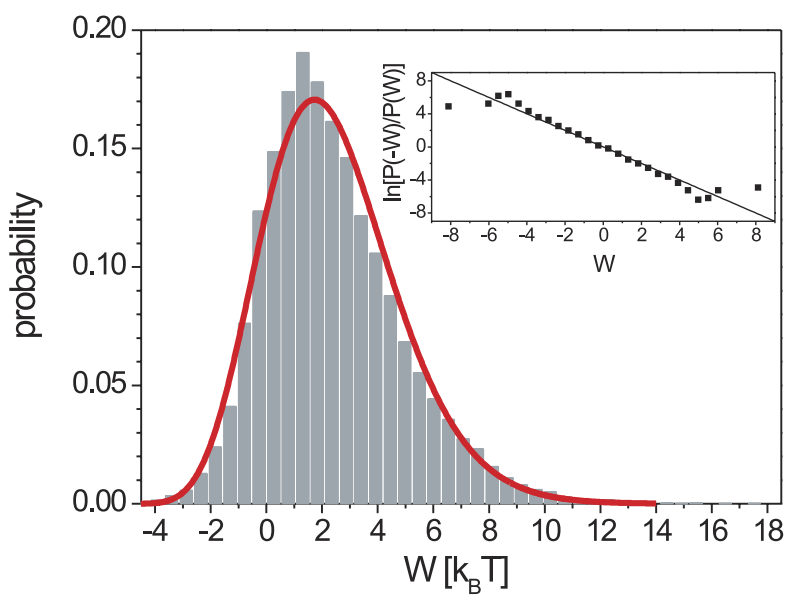

FIG. 4 (color online). Non-Gaussian work distribution. The data were taken from about 16000 trajectories, where the average work done on the particle was about $2.4 k_{\mathrm{B}} T$. The solid line shows the Pearson type III distribution [26] corresponding to the theoretically obtained moments. Inset: logarithm of the ratio of the probability to find trajectories with work $-W$ to those with work $+W$. The solid line shows the expected curve (9). The deviation is due to the poor statistics of large negative work values $W \lesssim-4 k_{\mathrm{B}} T$. 
TABLE I. Comparison between theoretically predicted and measured moments of the work probability based on the data shown in Fig. 4.

\begin{tabular}{lccc}
\hline \hline & $m_{1}\left[k_{\mathrm{B}} T\right]$ & $m_{2}\left[\left(k_{\mathrm{B}} T\right)^{2}\right]$ & $m_{3}\left[\left(k_{\mathrm{B}} T\right)^{3}\right]$ \\
\hline Experiment & 2.4 & 11.7 & 67.8 \\
Theory & 2.4 & 11.6 & 63.7 \\
\hline \hline
\end{tabular}

$$
\frac{\partial \rho}{\partial t}=\mathcal{L} \rho-\frac{\partial V}{\partial \tau} \frac{\partial \rho}{\partial w} .
$$

Here, $\rho(z, w, t)$ is the joint probability of the particle to be at time $t$ a distance $z$ away from the wall and to have accumulated an amount of work $w$ up to this time. The Fokker-Planck operator [23]

$$
\mathcal{L}=\frac{\partial}{\partial z}\left(\frac{D_{\perp}}{k_{\mathrm{B}} T} \frac{\partial V}{\partial z}+\frac{3}{2} \frac{\partial D_{\perp}}{\partial z}+D_{\perp} \frac{\partial}{\partial z}\right)
$$

governs the dynamics of the particle, where

$$
D_{\perp}(z) \approx D_{0}[1+R /(z-R)]^{-1}
$$

is the diffusion coefficient for perpendicular motion near a surface [24]. The free diffusion constant for a particle with radius $R=2 \mu \mathrm{m}$ is $D_{0} \simeq 0.1 \mu \mathrm{m}^{2} / \mathrm{s}$ at room temperature $T=293 \mathrm{~K}$. Since we start in equilibrium with no work spent on the particle yet, the initial distribution needed to solve Eq. (10) is

$$
\rho(z, w, 0)=\delta(w) \frac{\exp \left(-V(z, 0) / k_{\mathrm{B}} T\right)}{\int d z \exp \left(-V(z, 0) / k_{\mathrm{B}} T\right)} .
$$

Equation (10) is a Fokker-Planck equation in two space dimensions $z$ and $w$ including a singular initial condition, which numerically is not easy to handle. We therefore multiply Eq. (10) with $w^{n}$ and integrate over $w$. After one integration by parts we obtain the inhomogeneous evolution equation

$$
\frac{\partial M_{n}}{\partial t}=\mathcal{L} M_{n}+n \frac{\partial V}{\partial \tau} M_{n-1}
$$

for the conditional $n$th moment of the work

$$
M_{n}(z, t)=\int_{-\infty}^{+\infty} d w w^{n} \rho(z, w, t) .
$$

The actual $n$th moment $m_{n}$ then follows simply by integrating over $z$ and thus adding the contributions of all possible final positions of the particle. The function $M_{0}(z, t)$ is the probability distribution of the position $z$ of the particle and hence $m_{0}=1$. Table I compares the numerically and experimentally obtained first three moments of the data shown in Fig. 4. We stress that this good agreement does not involve a single fit parameter.

In summary, we have confirmed experimentally both a stochastic formulation of the first law and various recent theoretical ramifications of the second law in a timedependent nonharmonic potential, where the underlying distributions are typically non-Gaussian. In the next step, nonharmonic systems with broken detailed balance should be investigated to test theorems $[5,25]$ which, so far, have been under experimental scrutiny in the harmonic case only [11].

[1] K. Sekimoto, Prog. Theor. Phys. Suppl. 130, 17 (1998).

[2] D. J. Evans, E. G. D. Cohen, and G. P. Morriss, Phys. Rev. Lett. 71, 2401 (1993).

[3] G. Gallavotti and E. G. D. Cohen, Phys. Rev. Lett. 74, 2694 (1995).

[4] C. Jarzynski, Phys. Rev. Lett. 78, 2690 (1997).

[5] T. Hatano and S. Sasa, Phys. Rev. Lett. 86, 3463 (2001).

[6] C. Bustamante, J. Liphardt, and F. Ritort, Phys. Today 58, No. 7, 43 (2005).

[7] J. Liphardt, S. Dumont, S. B. Smith, I. Tinoco, Jr., and C. Bustamante, Science 296, 1832 (2002).

[8] D. Collin, F. Ritort, C. Jarzynski, S. Smith, I. Tinoco, and C. Bustamante, Nature (London) 437, 231 (2005).

[9] G. M. Wang, E. M. Sevick, E. Mittag, D. J. Searles, and D. J. Evans, Phys. Rev. Lett. 89, 050601 (2002).

[10] D. M. Carberry, J. C. Reid, G. M. Wang, E. M. Sevick, D. J. Searles, and D. J. Evans, Phys. Rev. Lett. 92, 140601 (2004).

[11] E. H. Trepagnier, C. Jarzynski, F. Ritort, G. E. Crooks, C. J. Bustamante, and J. Liphardt, Proc. Natl. Acad. Sci. U.S.A. 101, 15038 (2004).

[12] R. van Zon and E. G. D. Cohen, Phys. Rev. Lett. 91, 110601 (2003).

[13] T. Speck and U. Seifert, Phys. Rev. E 70, 066112 (2004).

[14] E. Cohen and D. Mauzerall, J. Stat. Mech.: Theor. Exp. P07006 (2004).

[15] C. Jarzynski, J. Stat. Mech.: Theor. Exp. P09005 (2004).

[16] D. Prieve, Adv. Colloid Interface Sci. 82, 93 (1999).

[17] Because of the characteristics of the electro-optical modulator the pulse can be parametrized as $I(\tau) \propto$ $\sin \left[A_{1} \cos \left(2 \pi \tau / t_{\mathrm{s}}\right)+\varphi\right]$.

[18] V. Blickle, D. Babic, and C. Bechinger, Appl. Phys. Lett. 87, 101102 (2005).

[19] The Rayleigh lengths of the upper and the lower optical tweezers is $\simeq 4.6 \mu \mathrm{m}$ and $425 \mu \mathrm{m}$, respectively; therefore, they produce no force gradients in $z$ direction on the range of the sampled particle positions.

[20] G. E. Crooks, Phys. Rev. E 61, 2361 (2000).

[21] S. Schuler, T. Speck, C. Tietz, J. Wrachtrup, and U. Seifert, Phys. Rev. Lett. 94, 180602 (2005).

[22] A. Imparato and L. Peliti, Europhys. Lett. 69, 643 (2005).

[23] H. Risken, The Fokker-Planck Equation (Springer-Verlag, Berlin, 1989), 2nd ed.

[24] H. Brenner, Chem. Eng. Sci. 16, 242 (1961).

[25] T. Speck and U. Seifert, J. Phys. A 38, L581 (2005).

[26] Handbook of Mathematical Functions, edited by M. Abramowitz and I. A. Stegun (Dover, New York, 1972), 9th ed., p. 930. 